\title{
Multistarter from organic viticulture for red wine Montepulciano d'Abruzzo production
}

\author{
Giovanna Suzzi ${ }^{1}$ *, Maria Schirone ${ }^{1}$, Manuel Sergi ${ }^{1}$, Rosa Maria Marianella ${ }^{2}$, Giuseppe Fasoli ${ }^{1}$, Irene Aguzzi ${ }^{1}$ \\ and Rosanna Tofalo ${ }^{1}$
}

${ }^{1}$ Department of Food Science, University of Teramo, Teramo, Italy

${ }^{2}$ Direzione Generale della Prevenzione e Repressione Frodi Roma, Ministero delle Politiche Agricole Alimentari e Forestali, Roma, Italy

\section{Edited by:}

Danilo Ercolini, Università degli Studi

di Napoli Federico II, Italy

\section{Reviewed by:}

Maurizio Ciani, Università Politecnica delle Marche, Italy

Sandra Torriani, Università degli Stud di Verona, Italy

*Correspondence:

Giovanna Suzzi, Department of Food Science, University of Teramo, 64023 Mosciano Sant'Angelo, Teramo, Italy. e-mail:gsuzzi@unite.it
In the last years the use of a multistarter fermentation process has been proposed to improve the organoleptic characteristics of wines. In the present study the fermentation performances and the interactions of mixed and sequential cultures of Hanseniaspora uvarum, Candida zemplinina, and a strain of Saccharomyces cerevisiae isolated from organic musts were investigated. To evaluate the oenological performances of the tested strains microvinifications in pasteurized red grape juice from Montepulciano d'Abruzzo cultivar were compared. The course of fermentation has been controlled through classical determinations $\left(\mathrm{CO}_{2}\right.$ evolution, ethanol, glycerol, $\mathrm{pH}$, total titratable acidity, sugar content, free sulfur dioxide $\left(\mathrm{SO}_{2}\right)$, dry extract, sugars, organic acids, and volatile compounds). Moreover, the yeast population was determined by both culture-dependent and independent approaches. In particular, the pure culture of $H$. uvarum and $C$. zemplinina did not end the fermentation. On the contrary, when $S$. cerevisiae was added, fermentations were faster confirming that yeast interactions influence the fermentation kinetics. Moreover, $C$. zemplinina showed a good interaction with $S$. cerevisiae by increasing the fermentation kinetic in high gravity Montepulciano must, with low ethyl acetate and acetic acid production. This study confirmed that non-Saccharomyces yeasts play a crucial role also in organic wines and their activity could be modulated through the selection of appropriate strains that correctly interact with $S$. cerevisiae.

Keywords: non-Saccharomyces wine yeast, mixed fermentation, metabolic profile, multistarter

\section{INTRODUCTION}

Over the last decade, the phenomenon of organic products has taken hold of large segments of consumers. At its most basic level, organic wine is made from grapes that have been grown with as little human impact as possible. Organic wine is a wine obtained from organically growing grapes without the help of or need for synthetic fertilizers, synthetic plant treatments, or herbicides (Trioli and Hofmann, 2009). Accurate studies have been carried out on soil and vineyard management in organic wine making whereas, as far as we know, no or few data are available on microbial populations of grape berries from organic vineyard as well as of those from organic wines. In a previous paper (Tofalo et al., 2011) the yeast populations present on grape berries and must from organic vineyards of red Montepulciano d'Abruzzo and white Trebbiano cultivars were studied. In particular non-Saccharomyces (NS) wine yeasts were identified at species level. Moreover the strains were typed and characterized for some oenological parameters. In recent years, a lot of studies evaluated the NS species present in wine ecosystem, and demonstrated the impact of grape conditions on NS populations (Fernández et al., 2000; Raspor et al., 2006; González et al., 2007). The role of NS yeasts in wine production has been debated extensively and several researchers have shown that NS yeasts survive during fermentation and could reach cell concentrations similar to those reached by Saccharomyces cerevisiae $10^{6}-10^{8}$ cells/ml (Fleet et al., 1984; Gafner and Schultz, 1996).
In fact, as suggested by several authors (Zironi et al., 1993; Gil et al., 1996; Lema et al., 1996; Toro and Vazquez, 2002; Ciani et al., 2006; Viana et al., 2008), there is growing evidence that NS yeasts play an important role in wine quality. Fleet (2008) discussed the possibilities of using yeasts other than those from the genus Saccharomyces for future wine fermentations and the commercial viability of mixed cultures, because NS species have great potential to introduce appealing characteristics to wine that may improve its organoleptic quality. Consequently, the impact of NS yeasts on wine fermentation cannot be ignored. The major NS yeasts present during organic must fermentation of Trebbiano and Montepulciano cultivars were Hanseniaspora uvarum, Metschnikowia fructicola and Candida zemplinina, representing 43, 31 and 11\%, respectively, of the total NS population isolated. Although the population size of these species was reduced throughout the wine fermentations, their growth was not completely suppressed and NS yeasts were still present at the end of the fermentation process (Tofalo et al., 2011). These yeasts from organic wine shared many characteristics which suggest that the strong selection pressure exerted by farming system and vine variety could have generated variability at different levels. Knowledge about the biodiversity of native yeasts is essential for the preservation and exploitation of the oenological potential of wine grape growing regions. The use of a selected multistarter (controlled mixed cultures) was proposed several years ago. In the middle of the last century, to reduce 
the acetic acid content of wine, Cantarelli (1955), Castelli (1969) encouraged the sequential use of Torulaspora delbrueckii (formerly known as Saccharomyces rosei) and S. cerevisiae. Later on, mixed cultures were proposed also for other objectives such as the biological deacidification of must or increase the glycerol content but one of the most investigated uses of mixed cultures relates to confer greater complexity to a wine, enhancing its organoleptic profile (Ciani et al., 2010). Several studies on mixed fermentations containing S. cerevisiae and NS wine yeasts have been carried out to evaluate the possibility of using controlled multistarter cultures to improve wine quality (Mora et al., 1990; Zironi et al., 1993; Toro and Vazquez, 2002; Ciani et al., 2006; Andorrà et al., 2010). For this purpose different NS species were used to study mixed fermentation such as Hanseniaspora guilliermondii, H. uvarum, Candida pulcherrima (Metschnikowia), Pichia kluyveri, Pichia fermentans, Candida cantarellii, T. delbrueckii, Kluyveromyces thermotolerans, Candida stellata (recently reclassified as Starmerella bombicola). The yeasts present on grape berries and must from organic vineyards could have a unique composition and these indigenous yeasts impart distinct regional and desired characteristics to wines. In this context, autochthonous NS strains could be selected to conferment organic musts alongside $S$. cerevisiae.

The aim of this research is to evaluate the fermentation performance and the interactions of mixed and sequential cultures of H. uvarum and C. zemplinina and a strain of S. cerevisiae isolated from organic must. The results of fermentation kinetics, secondary compound formation and sensorial analysis could be useful to formulate mixed starter cultures.

\section{MATERIALS AND METHODS YEAST STRAINS}

Non-Saccharomyces strains (C. zemplinina STS12 and H. uvarum STS45) have been isolated in a previous study from spontaneous fermentation of organic Montepulciano d'Abruzzo and Trebbiano grapes (Tofalo et al., 2011). All of the strains have been previously analyzed by sequencing the approximately $600 \mathrm{bp}$ D1/D2 region of the large (26S) ribosomal subunit using primers NL1 and NL4. These natural wine strains belong to the culture collection of the Food Science Department (University of Teramo, Italy). An autochthonous starter culture S. cerevisiae (STS1) was also used as starter culture (Tofalo et al., 2011). The strains were maintained at $-80^{\circ} \mathrm{C}$ in glycerol $20 \%(\mathrm{v} / \mathrm{v})$ and, in parallel, on agar slants under paraffin oil at $4^{\circ} \mathrm{C}$.

\section{MICROVINIFICATIONS}

To evaluate oenological performances, the strains were tested in microvinification trials using pasteurized must without grape skin from Montepulciano d'Abruzzo cultivar (280 g/l fermentable sugars, $7.4 \mathrm{~g} / \mathrm{l}$ titratable acidity (TTA) and a $\mathrm{pH} 3.2$ ). Fermentations were conducted using several combinations of the strain S. cerevisiae STS1 with strains C. zemplinina STS12 and H. uvarum STS45. The musts were inoculated with $10^{6}$ cells $/ \mathrm{ml}$ as follows: $S$. cerevisiae (S), C. zemplinina (C), H. uvarum $(\mathrm{H})$, C. zemplinina/S. cerevisiae (CS), H. uvarum/S. cerevisiae (HS), C. zemplinina/H. uvarum/S. cerevisiae (CHS), moreover the must was inoculated with all the three strains as follows C. zemplinina/H. uvarum/S. cerevisiae (RCHS) ratios of 25:25:50. A sequential fermentation
(QS) was inoculated with $10^{6}$ cells/ml C. zemplinina/H. uvarum and after $48 \mathrm{~h}$ S. cerevisiae was added for each strain.

The must samples $(95 \mathrm{ml})$ were inoculated with $5 \mathrm{ml}$ of a preculture grown for $48 \mathrm{~h}$ in the same must, as described by Tofalo et al. (2011). Fermentations were carried out in duplicate for each strain at a controlled temperature of $25^{\circ} \mathrm{C}$. The kinetic fermentations were monitored daily by gravimetric determinations, evaluating the loss of weight due to the production of $\mathrm{CO}_{2}$. When the $\mathrm{CO}_{2}$ evolution stopped (i.e., at constant weight), the samples were refrigerated for 2 days at $4^{\circ} \mathrm{C}$, racked, and stored at $-20^{\circ} \mathrm{C}$ until analysis. Non-inoculated must was used as negative control.

\section{DETERMINATION OF MICROBIAL GROWTH AND DIFFERENTIAL ENUMERATION}

From each flask, samples were taken along the fermentation process to evaluate viable cell counts of the inoculated species. One hundred microliters aliquots of serial dilutions of each sample were plated on Lysine Agar (LA medium; Oxoid Unipath, Hampshire, UK) and Wallerstein Laboratory nutrient agar medium (WLN medium; Oxoid; Pallmann et al., 2001) to estimate the NS yeast and the total yeast population, respectively. Moreover, a culture-independent approach was used. S. cerevisiae, H. uvarum, C. zemplinina specific quantitative PCR (qPCR) tests were carried out according Hierro et al. (2007) and Zott et al. (2010), respectively. DNA was extracted using the DNA PowerSoil ${ }^{\circledR}$ Isolation Kit (Mobio Laboratories, Inc.) according to the manufacturer's instructions.

Real-time amplifications were carried out in a $25 \mu 1$ reaction volume, using $1 \times 2$ XIQ SYBR Green PCR Supermix (Bio-Rad, Hercules, CA, USA), $0.2 \mu \mathrm{M}$ of each primer and $5 \mu \mathrm{l}$ of DNA suspension. All amplifications were carried out in optical-grade 96-well plates on a Cycle IQ system (Bio-Rad). The qPCR threshold cycle $\left(C_{\mathrm{t}}\right)$ was determined automatically by the instrument. Samples with known quantities of yeast cells were prepared to generate standard curves. Sterilized grape juice was inoculated with the yeast strains, plated on WLN media for viable counts. The counted samples were immediately extracted (triplicate), as described above. The DNA obtained was used to prepare serial dilutions, from $10^{8}$ to $10 \mathrm{cell} / \mathrm{ml}$. The correlation coefficient between $C_{\mathrm{t}}$ and count values was analyzed and interpreted using the appropriate Microsoft Excel function. Each $C_{\mathrm{t}}$ was the average of four measures obtained by amplifying four DNA extracts from the same artificially inoculated sample. In all PCR runs, negative controls (sterilized water), positive controls, and samples were run in triplicate. Sensitivity of qPCR assays was evaluated with reference to other reports (Hierro et al., 2006).

\section{PHYSICO-CHEMICAL DETERMINATIONS}

The main products (ethanol, glycerol, $\mathrm{pH}$, TTA, sugar content, free sulfur dioxide $\left(\mathrm{SO}_{2}\right)$ and dry extract of wine), and must under fermentation were determined on samples taken at the end of fermentation following the official International Organization of Vine and Wine (2011) methods of analysis. Organic acid, glucose, and fructose concentrations were determined according to Tofalo et al. (2011) and Lopez-Tamames et al. (1996), respectively. Biogenic amines production was determined according to Tofalo et al. (2007). All analyses were performed in triplicate. 


\section{SOLID PHASE MICROEXTRACTION-GAS CHROMATOGRAPHY ANALYSIS OF VOLATILE COMPOUNDS}

Five milliliters of wine samples were placed in $10 \mathrm{ml}$ glass vials with $1 \mathrm{~g} \mathrm{NaCl}$ and $10 \mu \mathrm{l}$ of 4-methyl-2-pentanol (final concentration $4 \mathrm{mg} / \mathrm{l}$ ) were added as internal standard. Both equilibration and adsorption phases were carried out by stirring for $30 \mathrm{~min}$ at $40^{\circ} \mathrm{C}$. A carboxen-polydimethylsiloxane-coated fiber $(85 \mu \mathrm{m})$ was used (Sigma-Aldrich, St. Louis, MO, USA). Under the extraction conditions described above, the recovery of the volatile compounds was between 88.9 and $103.5 \%$. For quantitative determination, a CP 380 capillary gas chromatograph equipped with a 8200 autosampler SPME III (Varian, Italy) was used. The fused silica capillary column was a CP-Wax $52 \mathrm{CB}(50 \mathrm{~m} \times 0.32 \mathrm{~mm})$ by Crompack (Netherlands), coated with polyethylene glycol (film thickness $1.2 \mu \mathrm{m})$, as stationary phase. The injector and FID temperature was $250^{\circ} \mathrm{C}$. After extraction, the fiber was placed in the injector of the GC for $15 \mathrm{~min}$. The temperature program was the following: initial temperature $\left(50^{\circ} \mathrm{C}\right)$ held for $2 \mathrm{~min}$; first ramp, $1^{\circ} \mathrm{C}$ min to $65^{\circ} \mathrm{C}$ ( 0 min hold $)$; second $\mathrm{ramp}, 10^{\circ} \mathrm{C} \mathrm{min}$ to $150^{\circ} \mathrm{C}(10 \mathrm{~min}$ hold $)$; third $\operatorname{ramp} 10^{\circ} \mathrm{C}$ min to $200^{\circ} \mathrm{C}(1 \mathrm{~min}$ hold $)$. The carrier gas $\left(\mathrm{N}_{2}\right)$ flow rate was $2.5 \mathrm{ml} / \mathrm{min}$. The aroma compounds were identified by comparing the retention time of standards and their identification was confirmed by using GC-MS. GC-MS analysis was performed using a GC-mass spectrometer Finnigan Trace DSQ Quadrupole (Thermo Finnigan, San Jose, CA, USA). Mass spectrometer conditions were: Ion Source: electron ionization (EI), Ion Polarity: POS, Ion Source Temperature: $250^{\circ} \mathrm{C}$, MS transfer line: $220^{\circ} \mathrm{C}$, Turbomolecular Pump: 70 l/s, Acquisition: full Scan, Mass range: $30-400 \mathrm{~m} / z$, Carrier gas: He. The data were processed using Xcalibur Data System Software 1.4.1 SP3 (Thermo Finnigan, San Jose, CA, USA). The quantitative analysis of wine aroma compounds was carried out on the basis of the relative peak area (Qi) calculated from head space SPME (HS/SPME) gas chromatograms after addition of know amounts of analyte standards, as well as the internal standard according to De la Calle-Garcia et al. (1998). The chemical analyses were carried out in the same period of the sensory analysis. Each determination was carried out in duplicate. The data presented are the means of three determinations. All reagents were purchased from Sigma-Aldrich (St. Louis, MO, USA), with a purity greater than $99 \%$.

\section{STATISTICAL ANALYSIS}

The mean and S.D. were calculated for each experimental parameter. Principal component analysis (PCA) was performed using statistical software STATISTICA for Windows (STAT. version 8.0, StatSoft Inc., Tulsa, OK, USA).

\section{RESULTS}

\section{COURSE OF FERMENTATION AND DEVELOPMENT OF YEASTS DURING FERMENTATIONS}

The course of fermentation rates with pure, mixed, and sequential cultures is given in Figure 1. The pure culture of $H$. uvarum was unable to finish fermentation according to the poor fermentative capacity of this species, but also the pure culture of C. zemplinina did not end the fermentation. The yeast interactions had a clear impact on the fermentation kinetics and the presence of $S$. cerevisiae gave faster fermentations.

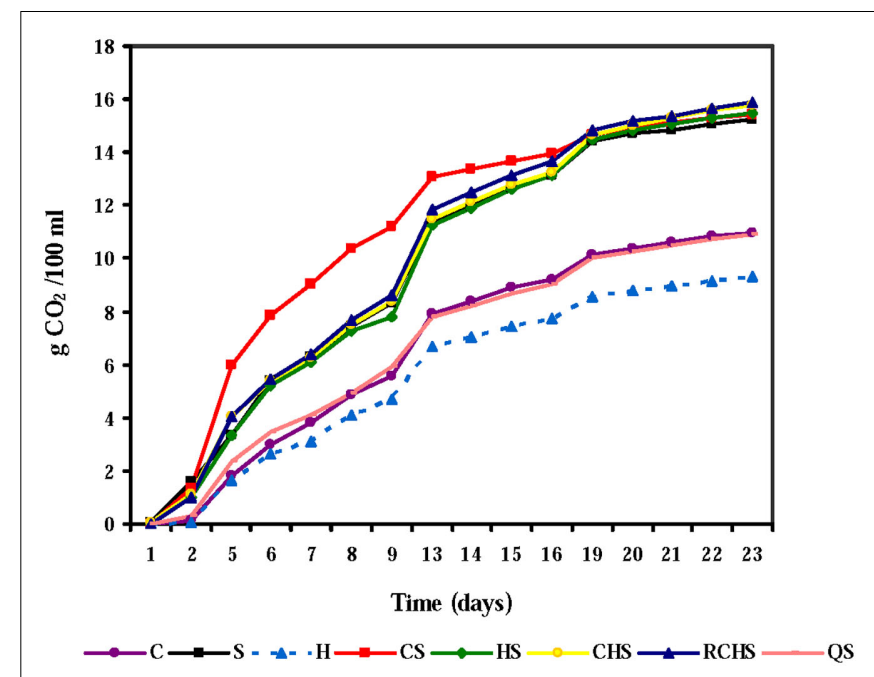

FIGURE 1 | Fermentation kinetics of pure, mixed, and sequential starter cultures in Montepulciano d'Abruzzo musts. C, C. zemplinina; S, S. cerevisiae; $H, H$. uvarum; CS, C. zemplinina/S. cerevisiae; $H S, H$. uvarum/S. cerevisiae; CHS, C. zemplinina/H. uvarum/S. cerevisiae; RCHS, C. zemplinina/H. uvarum/S. cerevisiae; QS, sequential fermentation.

In fact, fermentation kinetics of mixed cultures, HS, CHS, RCHS were comparable to those of S. cerevisiae pure culture. Only the trial CS showed a great improvement of fermentation kinetic during the first 15 days of fermentation highlighting the good association between C. zemplinina and S. cerevisiae. The RCHS trial, characterized by the inoculum of $C$. zemplinina and $H$. uvarum together with $S$. cerevisiae, showed a worst fermentation kinetic than CS trial probably for the lower proportion of cells of the two NS with respect to $S$. cerevisiae. The sequential trial QS presented a dramatic decrease of fermentation rate probably because $S$. cerevisiae was inoculated $48 \mathrm{~h}$ after the inoculum of C. zemplinina and H. uvarum.

The viable counts of yeast populations of the eight trials are reported in Table 1. The yeast populations of pure cultures of $S$. cerevisiae and C. zemplinina were similar, reaching maximum population around $10^{7} \mathrm{CFU} / \mathrm{ml}$ after $48 \mathrm{~h}$. In the case of $H$. uvarum pure culture the maximum population (around $10^{6} \mathrm{CFU} / \mathrm{ml}$ ) was reached after $48 \mathrm{~h}$, but starting from the $14^{\text {th }}$ day it started to decrease at $10^{5} \mathrm{CFU} / \mathrm{ml}$ and was not more countable on the $24^{\text {th }}$ day. Regarding the mixed fermentations HS and CS, NS yeasts were not found at the $14^{\text {th }}$ day, whereas S. cerevisiae delayed the reaching of maximum population. As regards the viable cells of the three species during mixed fermentation CHS and RCHS, differences were observed only in the latest one. In fact $H$. uvarum disappeared after $48 \mathrm{~h}$, whereas C. zemplinina increased up to $10^{5} \mathrm{CFU} / \mathrm{ml}$ and S. cerevisiae reached maximum population of about $10^{7} \mathrm{CFU} / \mathrm{ml}$ on the $14^{\text {th }}$ day. In general, S. cerevisiae quickly reached its maximum population and kept stable during the vinifications, with the exception of sequential fermentation QS during which S. cerevisiae was not able to grow after the $48 \mathrm{~h}$ growth of NS wine yeast. In fact, the number of viable cells of $S$. cerevisiae decreased up to $10^{5} \mathrm{CFU} / \mathrm{ml}$ at $24^{\text {th }}$ day. H. uvarum and C. zemplinina reached the maximum population of $10^{7} \mathrm{CFU} / \mathrm{ml}$ instead after $48 \mathrm{~h}$, but 
Table 1 | Yeast counts (log CFU/ml) and quantification by qPCR (in brackets) in pure, mixed, and sequential fermentation of organic Montepulciano d'Abruzzo musts.

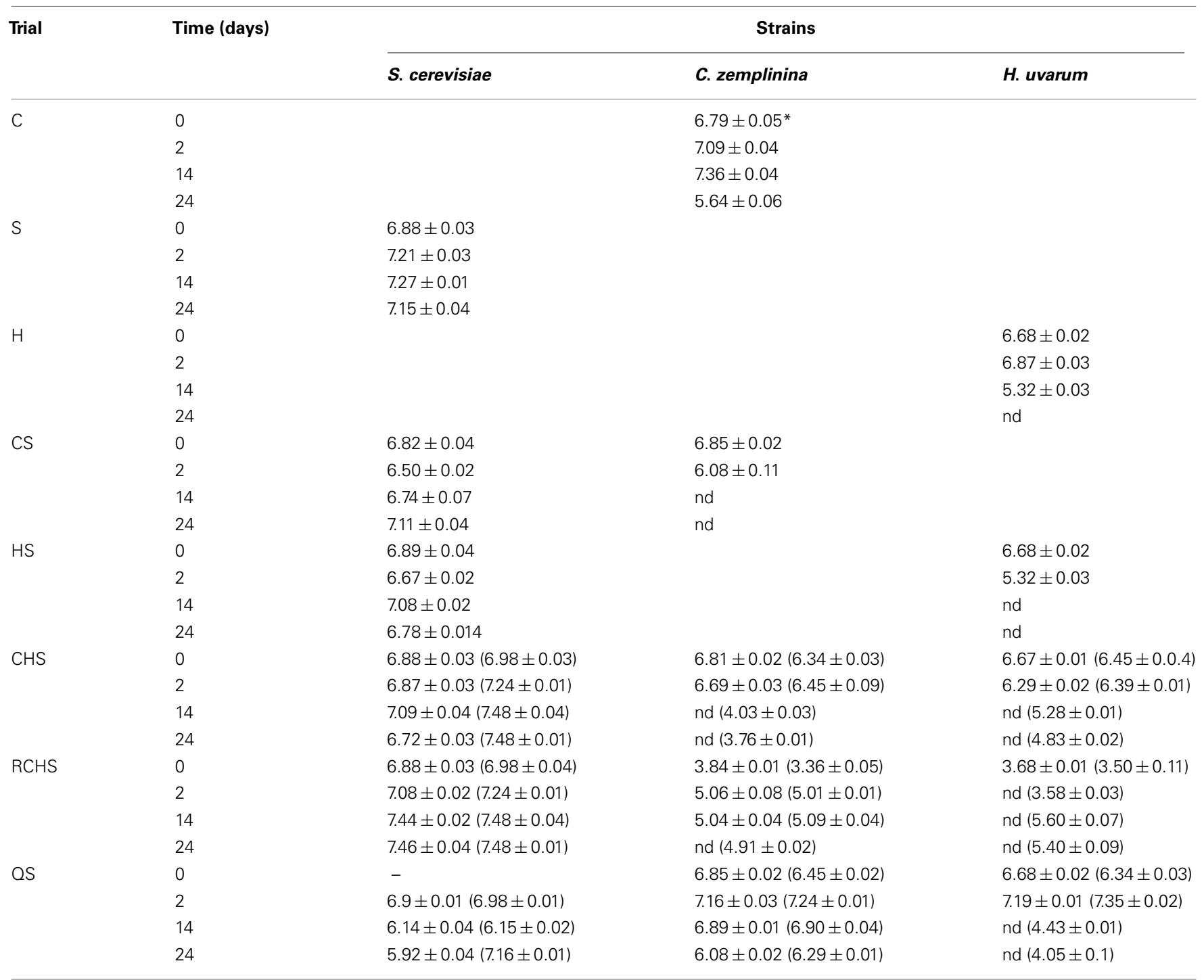

C, C. zemplinina; S, S. cerevisiae; H, H. uvarum; CS, C. zemplinina/S. cerevisiae; HS, H. uvarum/S. cerevisiae; CHS, C. zemplinina/H. uvarum/S. cerevisiae; RCHS, C. zemplinina/H. uvarum/S. cerevisiae; QS, Sequential Fermentation.

${ }^{*}$ Data are expressed as average $\pm S D ; n d$, not inoculated.

during the progress of fermentation the first one was not more countable whereas C. zemplinina decreased up to $10^{6} \mathrm{CFU} / \mathrm{ml}$.

\section{QUANTIFICATION OF MULTISTARTER POPULATION DURING FERMENTATION BY qPCR}

As culture-dependent techniques can underestimate the size and the diversity of a given population because they do not account for non-cultivable populations, the data on S. cerevisiae and NS populations during mixed fermentation obtained by plating were compared with those obtained by qPCR, as reported in Section "Materials and Methods." In particular the trials CHS, RCHS, and QS were analyzed by real-time PCR and the obtained results are reported in Table 1 . S. cerevisiae resulted to be always present at high level $\left(10^{6}-10^{7}\right.$ cells $\left./ \mathrm{ml}\right)$, but also H. uvarum and C. zemplinina were found at the end of fermentation, generally at about $10^{4}$ cells $/ \mathrm{ml}$. In particular H. uvarum at the end of fermentation of trail CHS showed a $C_{\mathrm{t}}$ value of 25 , corresponding to $10^{4}$ cells $/ \mathrm{ml}$ (data not shown).

\section{MAIN FERMENTATION PRODUCTS AND VOLATILE COMPOUNDS}

Table 2 reports some oenological parameters of the pure culture, mixed, and sequential fermentations. The pure cultures of $H$. uvarum and C. zemplinina did not finish the fermentations, leaving in the medium glucose and fructose, and only glucose, respectively. Also trial QS had $50 \mathrm{~g} / \mathrm{l}$ of residual glucose, as expected by the analysis of fermentation kinetics. The highest ethanol concentration was determined in pure culture of 
S. cerevisiae. All the multistarter fermentations reached a lower ethanol concentration ranging from 8.34 to $9.38 \%$. On the contrary the production of glycerol was greater by NS yeasts and in mixed cultures. Despite the high production of acetic acid in pure culture, $H$. uvarum did not increase volatile acidity in multistarter fermentations. No significant differences were found in the other compounds. Some discrepancies were found among ethanol, residual sugars, and other secondary metabolic compounds. What is stricking in our experiments was that all the strains showed an extremely poor ethanol yield from sugar consumed, which cannot be explained by the overproduction of any other metabolic products investigated in this study. Similar results have been previously obtained by Magyar and Toth (2011) and Tofalo et al. (2012).

Biogenic amines were detected at low levels in all eight microvinifications (Figure 2). In particular, the trial CS has the highest value of biogenic amines and QS has the lowest value. The pure cultures of $H$. uvarum, C. zemplinina, and $S$. cerevisiae did not produce cadaverine that was formed in all multistarter fermentations with the exception of QS. Tyramine,

Table 2 | Chemical profile of wines obtained with pure, mixed, and sequential starter cultures.

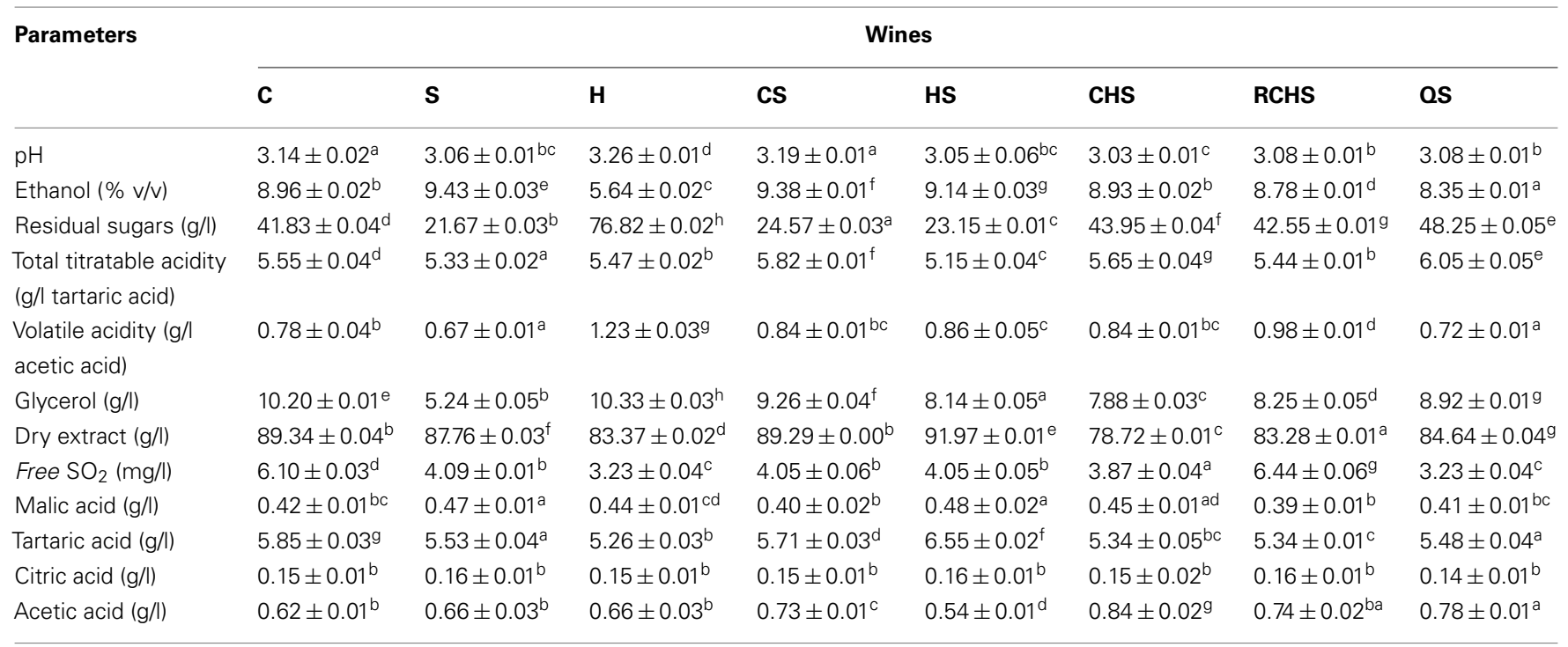

C, C. zemplinina; S, S. cerevisiae; H, H. uvarum; CS, C. zemplinina/S. cerevisiae; HS, H. uvarum/S. cerevisiae; CHS, C. zemplinina/H. uvarum/S. cerevisiae; RCHS, C. zemplinina/H. uvarum/S. cerevisiae; QS, Sequential Fermentation.

${ }^{*}$ Data are expressed as average $\pm S D$. Different letters $(a-h)$ in the same row correspond to statistically significant differences $(P<0.05)$.

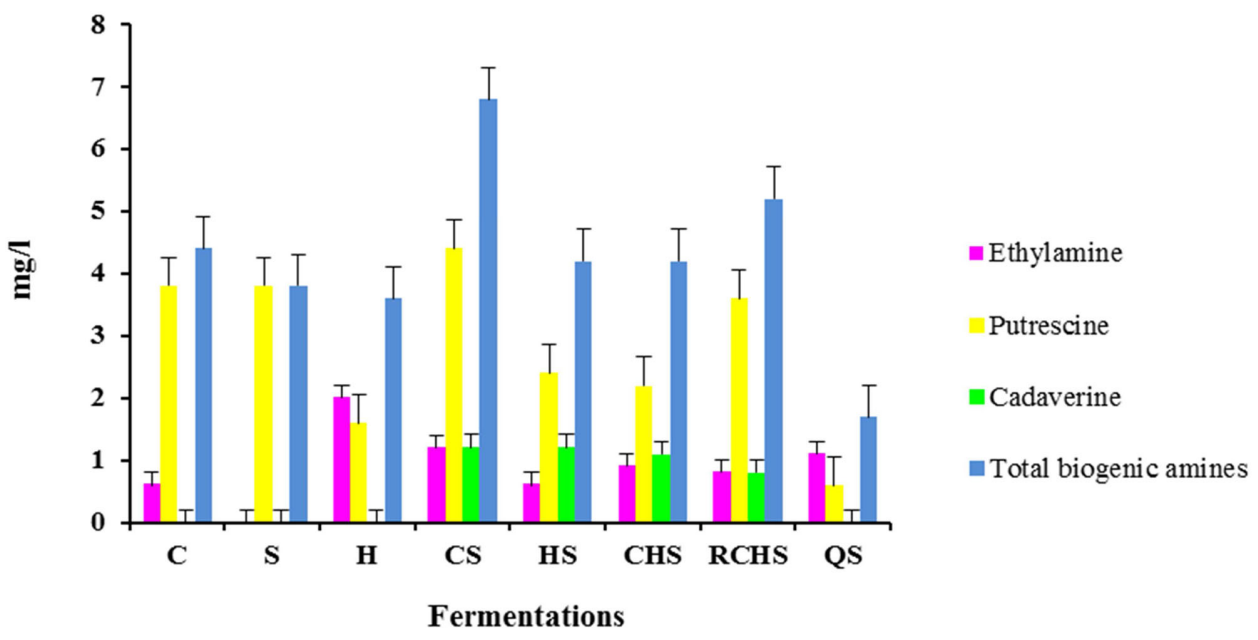

FIGURE 2 | Biogenic amines content in wines obtained with pure, mixed, and sequential starter cultures. $C, C$. zemplinina; $S, S$. cerevisiae; $H, H$. uvarum; CS, C. zemplinina/S. cerevisiae; $H S, H$.
uvarum/S. cerevisiae; CHS, C. zemplinina/H. uvarum/S. cerevisiae; RCHS, C. zemplinina/H. uvarum/S. cerevisiae; OS, sequential fermentation. 
histamine, 2-phenylethylamine, spermidine, and methylamine were not detectable in any the samples analyzed.

Table 3 shows some volatile compounds that well discriminated the aromatic profiles of the three wine yeast species. Pure cultures of C. zemplinina and S. cerevisiae produced low quantities of ethyl acetate and acetoin and high amounts of isoamyl alcohols and $\beta$-phenylethanol, having $C$. zemplinina the highest productions with respect to $S$. cerevisiae. In this study $H$. uvarum pure culture produced high amounts of ethyl acetate and acetoin and very low amounts of isoamyl alcohols and $\beta$-phenylethanol. The fermentations of mixed cultures CS, HS, and RCHS presented low levels of ethyl acetate and decreased the quantity of isoamylic alcohols and 2-phenylethanol with respect to $S$. cerevisiae. The fermentation of mixed culture CHS presented the highest formation of isoamyl alcohols and ethyl acetate. The sequential fermentation QS showed a completely different situation among the multistarter fermentations, with the lowest content of ethyl acetate and isoamyl alcohols and the highest one of acetoin, 2-phenylethanol, and isobutyl alcohol.

Principal component analysis has been used to obtain biochemical fingerprints of wines and to elucidate differences in the different components of wine fermented by different strains of Saccharomyces or by multistarter cultures (Nurgel et al., 2002; Howell et al., 2006).

Some compounds produced by the yeast pure and multistarter cultures were analyzed using PCA (Figures 3A,B). Firstly, the correlation matrix was computed in order to discriminate the variables, thus selecting 10 parameters (acetoin, 2,3 butanediol, ethanol, ethyl acetate, titratable and volatile acidity, glycerol, reducing sugars, phenylethyl alcohol and isoamyl alcohols). The PCA explained $70.6 \%$ of the total variance. PC 1 accounted for $53.55 \%$ of the variance and the negative segment of loading plot for this dimension (Figure 3B) was closely related to the levels of volatile acidity, whereas its positive counterpart was mainly related to ethanol. PC2 explained $17.05 \%$ of the variance; this dimension was mainly related positively with titratable acidity. Then, in score plot (Figure 3A) it is possible to distinguish three different groups of wines produced by strains. The fermentation carried out by $H$. uvarum was well differentiated from the others for the highest concentration of ethyl acetate, glycerol, acetoin, and volatile acidity; whereas C, CS, and CHS were very similar except for the concentration of isoamyl alcohols, 2,3 butanediol and reducing sugars (in $\mathrm{C}$ and CS, the values were higher than in CHS).

\section{DISCUSSION}

One of the most studied technologically advances in wine making is the inoculation of grape juice with mixed cultures of $S$. cerevisiae and NS yeasts. In this study the fermentation kinetics and metabolic compounds produced by multistarters during fermentation of organic musts were compared. Generally the fermentation kinetics of pure cultures of S. cerevisiae, H. uvarum, and $C$. zemplinina were in agreement with those expected and reported in other studies (Egli et al., 1998; Toro and Vazquez, 2002; Zohre and Erten, 2002; Mendoza et al., 2007; Fleet, 2008; Ciani et al., 2010). The pure culture of $H$. uvarum was unable to finish fermentation according to the poor fermentative capacity of this species, but also the pure culture of C. zemplinina did not end the fermentation. These two strains were selected on the basis of their fermentative capacity in a must containing $180 \mathrm{~g} / \mathrm{l}$ sugars (Tofalo et al., 2011) and probably the metabolic fermentative products from the high sugar Montepulciano must $(280 \mathrm{~g} / \mathrm{l})$ affected their performances. In particular, C. zemplinina has been reported to complete fermentation of Macabeo must containing $180 \mathrm{~g} / \mathrm{l}$ sugars (Andorrà et al., 2010) even if with a slight delay compared to the S. cerevisiae fermentation (Sipiczki et al., 2005; Tofalo et al., 2009, 2012). The yeast interactions had a clear impact on the fermentation kinetics and the presence of $S$. cerevisiae gave faster fermentations. However C. zemplinina and S. cerevisiae association (trial CS) showed a great improvement of kinetic during the first 15 days of fermentation. C. zemplinina is an osmotolerant and fructophilic yeast, generally producing low amounts of acetic acid and relevant quantities of glycerol from sugar fermentation

Table 3 | Aromatic compounds (mg/l) of the wines obtained with pure, mixed, and sequential starter cultures.

\begin{tabular}{|c|c|c|c|c|c|c|c|c|}
\hline \multirow{2}{*}{$\begin{array}{l}\text { Aromatic } \\
\text { compounds (\%) }\end{array}$} & \multicolumn{8}{|c|}{ Wines } \\
\hline & C & $\mathbf{S}$ & $\mathbf{H}$ & cs & HS & CHS & RCHS & os \\
\hline Ethyl acetate & $0.644 \pm 0.001^{a}$ & $1.850 \pm 0.003^{b}$ & $5.384 \pm 0.002^{c}$ & $0.441 \pm 0.003^{d}$ & $0.674 \pm 0.001^{\mathrm{e}}$ & $1.959 \pm 0.001^{f}$ & $0.511 \pm 0.003^{g}$ & $0.369 \pm 0.004^{h}$ \\
\hline Acetoin & $0.020 \pm 0.001^{d g}$ & $0.027 \pm 0.002^{h}$ & $0.533 \pm 0.002^{b}$ & $0.028 \pm 0.004^{h}$ & $0.075 \pm 0.001^{a}$ & $0.023 \pm 0.003^{h}$ & $0.014 \pm 0.004^{d}$ & $0.479 \pm 0.003^{e}$ \\
\hline Isoamyl alcohols & $8.673 \pm 0.003^{f}$ & $5.104 \pm 0.001^{e}$ & $1.237 \pm 0.004^{h}$ & $4.590 \pm 0.001^{a}$ & $3.750 \pm 0.001^{g}$ & $9.283 \pm 0.004^{c}$ & $5.174 \pm 0.004^{b}$ & $2.528 \pm 0.001^{d}$ \\
\hline 1-Hexanol & $0.031 \pm 0.0^{\mathrm{dg}}$ & $0.026 \pm 0.003^{\text {hd }}$ & $0.027 \pm 0.003^{\text {hd }}$ & $0.018 \pm 0.002^{\mathrm{e}}$ & $0.00 \pm 0.0^{\mathrm{a}}$ & $0.025 \pm 0.001^{h}$ & $0.00 \pm 0.0^{a}$ & $0.034 \pm 0.005^{g}$ \\
\hline 1-Octanol & $16.933 \pm 0.004^{f}$ & $0.170 \pm 0.003^{a}$ & $0.049 \pm 0.003^{h}$ & $0.085 \pm 0.002^{g}$ & $0.218 \pm 0.001^{e}$ & $0.043 \pm 0.003^{h}$ & $0.059 \pm 0.003^{d}$ & $0.407 \pm 0.003^{b}$ \\
\hline Phenylethyl alcohol & $2.928 \pm 0.004^{c}$ & $2.268 \pm 0.002^{b}$ & $0.00 \pm 0.00^{h}$ & $1.383 \pm 0.003^{d}$ & $1.859 \pm 0.002^{\mathrm{e}}$ & $1.469 \pm 0.003^{9}$ & $1.684 \pm 0.001^{a}$ & $2.394 \pm 0.002^{f}$ \\
\hline Isobutyl alcohol & $0.277 \pm 0.003^{\mathrm{e}}$ & $0.285 \pm 0.002^{b}$ & $0.219 \pm 0.001^{9}$ & $0.190 \pm 0.001^{d}$ & $0.663 \pm 0.002^{f}$ & $0.267 \pm 0.003^{a}$ & $0.156 \pm 0.001^{h}$ & $1.020 \pm 0.001^{c}$ \\
\hline
\end{tabular}

C, C. zemplinina; S, S. cerevisiae; H, H. uvarum; CS, C. zemplinina/S. cerevisiae; HS, H. uvarum/S. cerevisiae; CHS, C. zemplinina/H. uvarum/S. cerevisiae; RCHS, C. zemplinina/H. uvarum/S. cerevisiae; $Q S$, sequential fermentation.

Data are expressed as average $\pm S D$. Different letters $(a-h)$ in the same row correspond to statistically significant differences $(P<0.05)$. 

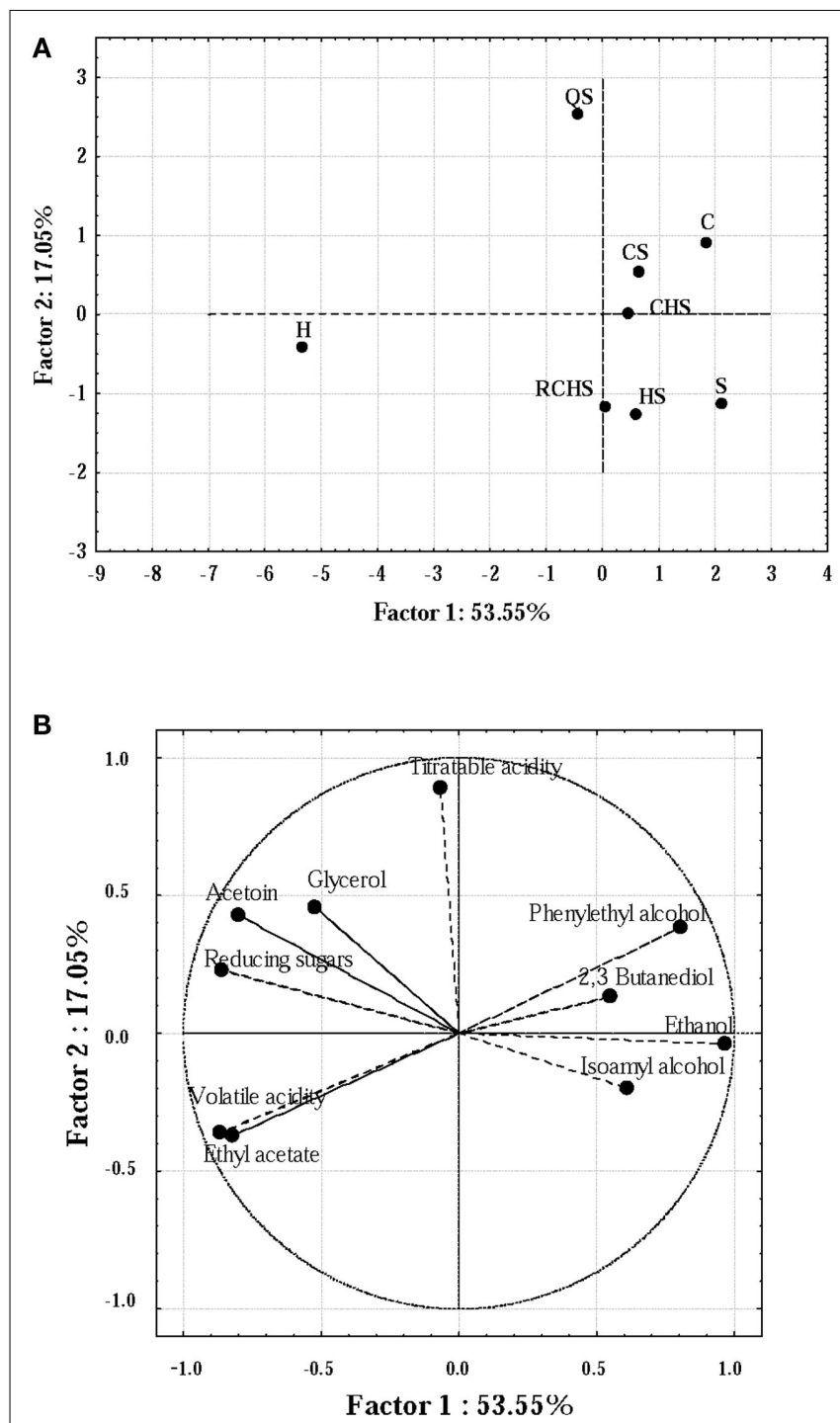

FIGURE 3 | Score plot (A) and loading plot (B) of the first and second principal components (PC) after PC analysis by the yeast pure and multistarter cultures. $C, C$. zemplinina; $S, S$. cerevisiae; $H, H$. uvarum; $C S$, C. zemplinina/S. cerevisiae; HS, H. uvarum/S. cerevisiae; CHS, C. zemplinina/H. uvarum/S. cerevisiae; RCHS, C. zemplinina/H. uvarum/S. cerevisiae; QS, sequential fermentation.

(Sipiczki et al., 2005; Magyar and Toth, 2011; Tofalo et al., 2011), it could be suggested that it will be able to consume sugars at the very beginning of the fermentation, alleviating the $S$. cerevisiae from the osmotic stress, thereby improving also the fermentation kinetics (Rantsiou et al., 2012). As previously demonstrated for S. cerevisiae species, different strains of C. zemplinina can have a specific effect, as reported by Tofalo et al. (2012). Moreover this positive interaction on fermentation kinetics between S. cerevisiae and C. zemplinina can have an important application on must with high sugar contents or special wines such as icewine, "passito," botrytized wines (Rantsiou et al., 2012). On the contrary the inoculum of C. zemplinina and H. uvarum together with $S$. cerevisiae (RCHS) did not produce the same results of CS trial, probably for the lower proportion of cells of the two NS with respect to S. cerevisiae.

The inoculum of S. cerevisiae after $48 \mathrm{~h}$ since that of C. zemplinina and H. uvarum (trial QS) produced a stuck fermentation Similar results have been reported in other studies, even if the mechanisms of this performance reduction of S. cerevisiae have not yet been explained. When S. cerevisiae and C. cantarelli interact during fermentation, the maximum S. cerevisiae population decreases (Toro and Vazquez, 2002). This fact may be due to amino acid and vitamin consumption during the first days of fermentation that can disable the subsequent growth and fermentative capacity of S. cerevisiae (Fleet, 2003). On the other hand these data were confirmed by the plating counts. S. cerevisiae dominated all the multistarter fermentations a part trial QS in which C. zemplinina had its best performance. The higher ability of S. cerevisiae to withstand the stress conditions such as increasing ethanol, decreasing $\mathrm{pH}$, nutrition depletion is generally considered to drive the wine yeast population dynamics (Pretorius, 2000). These selective pressures are currently questioned, whereas cell-cell interactions are being put forward as significant in affecting yeast succession (Ciani and Pepe, 2002; Fleet, 2003; Nissen et al., 2003). The early death of C. zemplinina and H. uvarum during mixed fermentation, even if with quantitative differences, appeared to be due to the antagonistic effect of S. cerevisiae as reported also by Andorrà et al. (2010) in the same species. The NS, in mixed fermentations, decreased as fermentation proceeded. On the contrary other authors (Ciani et al., 2006; Mendoza et al., 2007) reported that the presence of both Saccharomyces and NS yeasts promotes an increase in the persistence of NS yeasts during fermentation process. However it is clear that $S$. cerevisiae has a relevant antagonist effect upon C. zemplinina and $H$. uvarum, but also that the same NS can affect $S$. cerevisiae, depending on the sequence of growth, the number of viable cells and strain specificity. Few studies have been carried out to elucidate the mechanisms of these antagonistic phenomena (Nissen et al., 2003; Arneborg et al., 2005; Pérez-Nevado et al., 2006). During alcoholic fermentation yeasts can produce compounds that can have inhibitory effects against other yeast species or strains, such as short to mediumchain fatty acids (Ludovico et al., 2001; Fleet, 2003), killer toxins (Schmitt and Breinig, 2002), growth and nutritional conditions (Fleet, 2003). However in the above-mentioned studies, cell-cell contact-mediated mechanism appear to effect antagonism among yeasts. To confirm the data obtained by plating counts qPCR was used as culture-independent method. In fact sub lethally injured and/or viable but non-culturable (VBNC) cells, may fail to grow on plates and are common in wine (Millet and Lonvaud-Funel, 2000; Andorrà et al., 2011). The qPCR method used was previously developed to monitor the yeast evolution during alcoholic fermentations (Hierro et al., 2007; Andorrà et al., 2011). However, qPCR targeted at DNA quantifies also dead yeasts because of the DNA's stability (Hierro et al., 2006). Our data confirmed those of Andorrà et al. (2011) who found high populations of H. uvarum (up to $10^{8}$ cells $/ \mathrm{ml}$ ) throughout the mixed fermentations by qPCR methods. Of course these findings do not resolve the question if these populations correspond to VBNC, injured and/or dead cells or how many of these cells are metabolically active and how these in turn can influence the final wines (Andorrà et al., 2011). This 
aspect needs further researches and advances. When some yeasts develop together under fermentation conditions, they do not passively coexist, but rather they interact and can produce different levels of fermentation products, which can affect the chemical and aromatic composition of wines (Howell et al., 2006; Anfang et al., 2009). Grape must fermentations performed by pure, mixed or sequential cultures of NS yeasts with $S$. cerevisiae can produce wines with significant differences in the chemical composition (Herraiz et al., 1990; Ciani and Picciotti, 1995; Gil et al., 1996; Lambrechts and Pretorius, 2000; Rojas et al., 2003; Romano et al., 2003; Moreira et al., 2008). A relevant consequence of the NS yeasts was the increased presence of glycerol in all the multistarter fermentations, due the intrinsic characteristic of C. zemplinina and H. uvarum. In similar way acetic acid production is considered as a common pattern in apiculate yeasts that for this reason have been considered for long time as spoilage yeasts (Romano et al., 2003). Despite the high production of acetic acid in pure culture, H. uvarum did not increase volatile acidity in multistarter fermentations. These results are in complete agreement with those reported by other authors (Ciani et al., 2006; Mendoza et al., 2007; Andorrà et al., 2010). As regards biogenic amines, few studies have been conducted on their formation by yeasts, comparing different yeast species and quantifying only histamine (Torrea and Ancín, 2002). Their presence in wine ranged from a few milligrams per liter to about $50 \mathrm{mg} / \mathrm{l}$ depending on the wine. This study confirmed the low amino-decarboxylase of wine yeasts, even if some differences were determined in the eight trials. Even if a number of authors suggest that yeasts do not appear to be responsible for the production of most amines found in industrial commercial red wines (Marcobal et al., 2006; Smit et al., 2008), the yeast contribution to biogenic amine production could therefore be indirect: yeasts can alter the composition of grape musts by using some amino acids and secreting others during alcoholic fermentation and autolysis, thereby changing the concentration of precursor amino acids in the wine that can be used by other microorganisms in subsequent fermentation steps (Soufleros et al., 1998). According to the intrinsic characteristic of the single species, the production of main aromatic compounds was well differentiated in the trials. Low quantities of ethyl acetate and acetoin and high amounts of isoamyl alcohols and $\beta$-phenylethanol were produced by pure cultures of $C$. zemplinina and $S$. cerevisiae, as similarly reported by Andorrà et al. (2010). In this study H. uvarum pure culture produced high amounts of ethyl acetate and acetoin and very low amounts of isoamyl alcohols and $\beta$-phenylethanol. The production of large quantities of ethyl acetate and acetic acid by H. uvarum has always been considered a negative characteristic

\section{REFERENCES}

Andorrà, I., Berradre, M., Rozès, N., Mas, A., Guillamón, J. M., and Esteve-Zarzoso, B. (2010). Effect of pure and mixed cultures of the main wine yeast species on grape must fermentations. Eur. Food Res. Technol. 231, 215-224.

Andorrà, I., Monteiro, M., EsteveZarzoso, B., Albergaria, H., and Mas, A. (2011). Analysis and direct quantification of Saccharomyces cerevisiae and Hanseniaspora guilliermondii populations during alcoholic fermentation by fluorescence in situ hybridization, flow cytometry and quantitative PCR. Food Microbiol. 28, 1483-1491.

Anfang, N., Brajkovich, M., and Goddard, M. R. (2009). Co-fermentation with Pichia kluyveri increases varietal thiol concentrations in

(Ciani and Picciotti, 1995), whereas there is controversy concerning the production of higher alcohols. H. uvarum and K. apiculata were found to be the main producers of higher alcohols by Gil et al. (1996). However, some authors (Herraiz et al., 1990; Rojas et al., 2003; Romano et al., 2003) reported that apiculate yeasts were low producers of higher alcohols and can promote the esterification of various alcohols such as ethanol, geraniol, isoamyl alcohols, and 2-phenylethanol. Probably high production of higher alcohols could be a strain character dependent also in some NS wine yeasts (Capece et al., 2005). The secondary volatile compounds produced by mixed cultures were a combination of the different strains. PCA well highlighted these differences, indicating that the final wine characteristics can be modulated by different combinations or species and sequence of inoculum (Nurgel et al., 2002; Howell et al., 2006).

Candida zemplinina showed interesting features for mixed fermentation with $S$. cerevisiae, in particularly increasing the fermentation kinetic in high gravity Montepulciano must, with low ethyl acetate and acetic acid production. The combined use of three starter cultures (CSH and $\mathrm{RCSH}$ ) could allow the improvement of the organoleptic characteristics of a wine. Further studies are needed to clarify the interaction among the different starters and to optimize the fermentation and the modalities of inoculation. Effectively the use of NS wine yeasts together with Saccharomyces strains in mixed fermentations might be recommended as a tool to obtain the advantages of spontaneous fermentation of organic wines such as those obtained with Montepulciano, while avoiding the risks of stuck fermentation (Rojas et al., 2003; Romano et al., 2003; Jolly et al., 2006; Ciani et al., 2010). Because of the NS yeast strains biodiversity about their production level of enzymatic activities (Manzanares et al., 1999, 2000; Mendes-Ferreira et al., 2001; Strauss et al., 2001) and fermentation metabolites (Romano et al., 1992, 2003; Capece et al., 2005) of enological importance, suitable strains should be selected in order to be able to design mixed starter able to provide beneficial contributions also for wine obtained by organic viticulture. In particular C. zemplinina showed a good interaction with $S$. cerevisiae by increasing the fermentation kinetic in high gravity Montepulciano must, with low ethyl acetate and acetic acid production.

\section{ACKNOWLEDGMENTS}

This work is part of the project "Oenological microbiota: selection to identify the wine character and to improve the competitiveness of Montepulciano d'Abruzzo wineries" supported by grant from Cassa di Risparmio di Teramo.

Sauvignon Blanc. Aust. J. Grape Wine Res. 15, 1-8.

Arneborg, N., Siegumfeldt, H., Andersen, G., Nissen, P., Daria, V., Rodrigo, P., and Gluckstad, J. (2005). Interactive optical trapping shows that confinement is a determinant of growth in a mixed yeast. FEMS Microbiol. Lett. 245, 155-159.

Cantarelli, C. (1955). Studio comparativo dei lieviti apiculati dei generi
Kloeckera (Janke) ed Hanseniaspora (Zikes). Ann. Microbiol. 6, 85.

Capece, A., Fiore, C., Maraz, A., and Romano, P. (2005). Molecular and technological approaches to evaluate strain biodiversity in Hanseniaspora uvarum of wine origin. J. Appl. Microbiol. 98, 136-144.

Castelli, T. (1969). Vino al microscopio. Roma: Scialpi Editore. 
Ciani, M., Beco, L., and Comitini, F. (2006). Fermentation behaviour and metabolic interactions of multistarter wine yeast fermentations. Int. J. Food Microbiol. 108, 239-245.

Ciani, M., Comitini, F., Mannazzu, I., and Domizio, P. (2010). Controlled mixed culture fermentation: a new perspective on the use of nonSaccharomyces yeasts in winemaking. FEMS Yeast Res. 10, 123-133.

Ciani, M., and Pepe, V. (2002). The influence of pre-fermentative practices on the dominance of inoculated yeast starter under industrial conditions. J. Sci. Food Agric. 82, 573-578.

Ciani, M., and Picciotti, G. (1995). The growth kinetics and fermentation behaviour of some nonSaccharomyces yeasts associated with wine-making. Biotechnol. Lett. 17, 1247-1250.

De la Calle-Garcia, D., Reichenbacher, M., Dancer, K., Hurlbeck, C., Bartzsch, C., and Feller, K. H. (1998). Analysis of wine bouquet components using headspace space solidphase microextraction-capillary gas chromatography. J. High Resolut. Chromatogr. 21, 373-377.

Egli, C. M., Edinger, W. D., Mitrakul, C. M., and Henick-Kling, T. (1998). Dynamics of indigenous and inoculated yeast populations and their effect on the sensory character of riesling and Chardonnay wines. J. Appl. Microbiol. 85, 779-789.

Fernández, M. T., Ubeda, J. F., and Briones, A. I. (2000). Typing of nonSaccharomyces yeasts with enzymatic activities of interest in winemaking. Int. J. Food Microbiol. 59, 29-36.

Fleet, G. H. (2003). Yeast interactions and wine flavour. Int. J. Food Microbiol. 86, 11-22.

Fleet, G. H. (2008). Wine yeast for the future. FEMS Yeast Res. 8, 979-995.

Fleet, G. H., Lafon-Lafourcade, S., and Ribéreau-Gayon, P. (1984). Evolution of yeasts and lactic acid bacteria during fermentation and storage of Bordeaux Wines. Appl. Environ. Microbiol. 48, 1034-1038.

Gafner, J., and Schultz, M. (1996). Impact of glucose-fructose ratio on stuck fermentations: practical experiences to restart stuck fermentation. Vitic. Enol. Sci. 51, 214-218.

Gil, J., Mateo, J., Jiménez, M., Pastor, A., and Huerta, T. (1996). Aroma compounds in wine as influenced by apiculate yeasts. J. Food Sci. 61, 1247-1266.

González, S. S., Barrio, E., and Querol,A. (2007). Molecular identification and characterization of wine yeast isolated from Tenerife (Canary Island,
Spain). J. Appl. Microbiol. 102, 1018-1025.

Herraiz, T., Reglero, G., Herraiz, M., Martín-Alvarez, P., and Cabezudo, M. D. (1990). The influence of the yeast and type of culture on the volatile composition of wines fermented without sulfur dioxide. Am. J. Enol. Vitic. 41, 313-318.

Hierro, N., Esteve-Zarzoso, B., González, A., Mas, A., and Guillamón, J. M. (2006). Real-time quantitative PCR (QPCR) and reverse transcription-QPCR for detection and enumeration of total yeasts in wine. Appl. Environ. Microbiol. 72, 7148-7155.

Hierro, N., Esteve-Zarzoso, B., Mas, A., and Guillamón, J. M. (2007). Monitoring of Saccharomyces and Hanseniaspora populations during alcoholic fermentation by real-time quantitative PCR. FEMS Yeast Res. 7, 1340-1349.

Howell, K. S., Cozzolino, D., Bartowsky, E. J., Fleet, G. H., and Henschke, P. A. (2006). Metabolic profiling as a tool for revealing Saccharomyces interactions during wine fermentation. FEMS Yeast Res. 6, 91-101.

International Organization of Vine and Wine. (2011). Compendium of International Methods of Wine and must Analysis.

Jolly, N. P., Augustyn, O. P. H., and Pretorius, I. S. (2006). The role and use of non-Saccharomyces yeasts in wine production. S. Afr. J. Enol. Vitic. 27, 15-39.

Lambrechts, M. G., and Pretorius, I. S. (2000). Yeast and its importance to wine aroma. A review. South Afr. J. Enol. Vitic. 21, 97-129.

Lema, C., Garcia-Jares, C., Orriols, I., and Angulo, L. (1996). Contribution of Saccharomyces and nonSaccharomyces populations to the production of some components of Albarino wine aroma. Am. J. Enol. Vitic. 47, 206-216.

Lopez-Tamames, E., Puig-Deu, M., Teixeira, E., and Buxaderas, S. (1996). Organic acids, sugars, and glycerol content in white winemaking products determined by HPLC: relationship to climate and varietal factors. Am. J. Enol. Vitic. 47, 193-198.

Ludovico, P., Sousa, M. J., Silva, M. T., Leao, C., and Corte-Real, M. (2001). Saccharomyces cerevisiae commits to a programmed cell death process in response to acetic acid. Microbiology 147, 2409-2415.

Magyar, I., and Toth, T. (2011). Comparative evaluation of some oenological properties in wine strains of Candida stellata, Candida zemplinina,
Saccharomyces uvarum and Saccharomyces cerevisiae. Food Microbiol. 28, 94-100.

Manzanares, P., Ramon, D., and Querol, A. (1999). Screening of nonSaccharomyces wine yeasts for the production of $\beta$-D-xylosidase activity. Int. J. Food Microbiol. 46, 105-112.

Manzanares, P., Rojas, V., Genovés, S., and Vallés, S. (2000). A preliminary search for anthocyanin$\beta$-D-glucosidase activity in nonSaccharomyces wine yeasts. Int. J. Food Sci. Technol. 35, 95-103.

Marcobal, Á., Martín-Álvarez, P. J., Polo, C., Muñoz, R., and Moreno-Arribas, M. V. (2006). Formation of biogenic amines throughout the industrial manufacture of red wine. J. Food Prot. 69, 397-404.

Mendes-Ferreira, A., Climaco, M. C. and Mendes-Faia, A. (2001). The role of non-Saccharomyces species in releasing glycosidic bound fraction of grape aroma components - a preliminary study. J. Appl. Microbiol. 91, 67-71.

Mendoza, L. M., Manca de Nadra, M. C., and Farías, M. E. (2007). Kinetics and metabolic behavior of a composite culture of Kloeckera apiculato and Saccharomyces cerevisiae wine related strains. Biotechnol. Lett. 29, 1057-1063.

Millet, V., and Lonvaud-Funel, A. (2000). The viable but non-cultivable state of wine microorganisms during storage. Lett. Appl. Microbiol. 30, 136-141.

Mora, J., Barbas, J. I., and Mulet, A. (1990). Growth of yeast species during the fermentation of musts inoculated with Kluyveromyces thermotolerans and Saccharomyces cerevisiae. Am. J. Enol. Vitic. 41, 156-159.

Moreira, N., Mendes, F., Guedes de Pinho, P., Hogg, T., and Vasconcelos, I. (2008). Heavy sulphur compounds, higher alcohols and esters production profile of Hanseniaspora uvarum and Hanseniaspora guilliermondii grown as pure and mixed cultures in grape must. Int. J. Food Microbiol. 124, 231-238.

Nissen, P., Nielsen, D., and Arneborg, N. (2003). Saccharomyces cerevisiae cells at high concentrations cause early growth arrest of nonSaccharomyces yeasts in mixed cultures by a cell-cell contact-mediated mechanism. Yeast 20, 331-341.

Nurgel, C., Erten, H., Canbas, A. Cabaroglu, T., and Selli, S. (2002). Influence of Saccharomyces cerevisiae strains on fermentation and flavor compounds of white wines made from cv. Emir grown in Central
Anatolia, Turkey. J. Ind. Microbiol. Biotechnol. 29, 28-33.

Pallmann, C. L., Brown, J. A., Olineka, T. L., Cocolin, L., Mills, D. A., and Bisson, L. F. (2001). Use of WL medium to profile native flora fermentations. Am. J. Enol. Vitic. 52, 198-203.

Pérez-Nevado, F., Albergaria, H., Hogg, T., and Girio, F. (2006). Cellular death of two non-Saccharomyces wine-related yeasts during mixed fermentation with Saccharomyces cerevisiae. Int. J. Food Microbiol. 108, 336-345.

Pretorius, I. S. (2000). Tailoring wine yeast for the new millennium: novel approaches to the ancient art of winemaking. Yeast 16, 675-729.

Rantsiou, K., Dolci, P., Giacosa, S., Torchio, F., Tofalo, R., Torriani, S., Suzzi, G., Rolle, L., and Cocolin, L. (2012) Candida zemplinina can reduce acetic acid production by Saccharomyces cerevisiae in sweet wine fermentations. Appl. Environ. Microbiol. 78, 1987-1994.

Raspor, P., Milek, D. M., Polanc, J., Mozina, S. S., and Cadez, N. (2006). Yeasts isolated from three varieties of grapes cultivated in different locations of the Dolenjska vine-growing region, Slovenia. Int. J. Food Microbiol. 109, 97-102.

Rojas, V., Gil, J., Piñaga, F., and Manzanares, P. (2003). Acetate ester formation in wine by mixed cultures in laboratory fermentations. Int. J. Food Microbiol. 86, 181-188.

Romano, P., Fiore, C., Paraggio, M., Caruso, M., and Capece, A. (2003). Function of yeasts species and strains in wine flavour. Int. J. Food Microbiol. 86, 169-180.

Romano, P., Suzzi, G., Comi, G., and Zironi, R. (1992). Higher alcohol and acetic acid production by apiculate wine yeasts. J. Appl. Bacteriol. 73, 126-130.

Schmitt, M. J., and Breinig, F. (2002). The viral killer system in yeast: from molecular biology to application. FEMS Microbiol. Rev. 26, 257-276.

Sipiczki, M., Ciani, M., and Csoma, H. (2005). Taxonomic reclassification of Candida stellata DBVPG 3827. Folia Microbiol. (Praha) 50, 494-498.

Smit, A. Y., du Toit, W. J., and du Toit, M. (2008). Biogenic amines in wine: understanding the headache. South Afr. J. Enol. Vitic. 29, 109-127.

Soufleros, E., Barrios, M. L., and Bertrand, A. (1998). Correlation between the content of biogenic amines and other wine compounds. Am. J. Enol. Vitic. 49, 266-269.

Strauss, M. L. A., Jolly, N. P., Lambrechts, M. G., and van Rensburg, P. (2001). Screening for the production 
of extracellular hydrolytic enzymes by non-Saccharomyces wine yeasts. J. Appl. Microbiol. 91, 182-190.

Tofalo, R., Chaves-López, C., Di Fabio, F., Schirone, M., Felis, G. E., Torriani, S., Paparella, A., and Suzzi, G. (2009). Molecular identification and osmotolerant profile of wine yeasts that ferment a high sugar grape must. Int. J. Food Microbiol. 130, 179-187.

Tofalo, R., Schirone, M., Telera, G. C., Manetta, A. C., Corsetti, A., and Suzzi, G. (2011). Influence of organic viticulture on nonSaccharomyces wine yeast populations. Ann. Microbiol. 61, 57-66.

Tofalo, R., Schirone, M., Torriani, S., Rantsiou, K., Cocolin, L., Perpetuini, G., and Suzzi, G. (2012). Diversity of Candida zemplinina strains from grapes and Italian wines. Food Microbiol. 29, 18-26.

Tofalo, R., Torriani, S., Chaves-López, C., Martuscelli, M., Paparella, A., and Suzzi, G. (2007). A survey of Saccharomyces populations associated with wine fermentations from the Apulia region (South Italy). Ann. Microbiol. 57, 545-552.

Toro, M. E., and Vazquez, F. (2002). Fermentation behavior of controlled mixed and sequential cultures of Candida cantarellii and Saccharomyces cerevisiae wine yeasts. World J. Microbiol. Biotechnol. 18, 347-354.

Torrea, D., and Ancín, C. (2002). Content of biogenic amines in a Chardonnay wine obtained through spontaneous and inoculated fermentation. J. Agric. Food Chem. 50, 4895-4899.

Trioli, G., and Hofmann, U. (2009). ORWINE: Code of Good Organic Viticulture and Wine-Making. Oppenheim: ECOVIN-Federal Association of Organic Wine-Producer.

Viana, F., Gil, J. V., Genovés, S., Vallés, S., and Manzanares, P. (2008). Rational selection of non-Saccharomyces wine yeasts for mixed starters based on ester formation and enological traits. Food Microbiol. 25 778-785.

Zironi, R., Romano, P., Suzzi, G., Battistuta, F., and Comi, G. (1993). Volatile metabolites produced in wine by mixed and sequential cultures of Hanseniaspora guilliermondii or Kloeckera apiculata and Saccharomyces cerevisiae. Biotechnol. Lett. 15, 235-238.

Zohre, D. E., and Erten, H. (2002). The influence of Kloeckera apiculata and Candida pulcherrima yeasts on wine fermentation. Process. Biochem. 38, 319-324.

Zott, K., Claisse, O., Lucas, P., Coulon, J., Lonvaud-Funel, A., and MasneufPomarede, I. (2010). Characterization of the yeast ecosystem in grape must and wine using real-timePCR. Food Microbiol. 27, 559-567.

Conflict of Interest Statement: The authors declare that the research was conducted in the absence of any commercial or financial relationships that could be construed as a potential conflict of interest.

Received: 09 January 2012; accepted: 21 March 2012; published online: 18 April 2012.

Citation: Suzzi G, Schirone $M$, Sergi $M$, Marianella RM, Fasoli G, Aguzzi I and Tofalo R (2012) Multistarter from organic viticulture for red wine Montepulciano d'Abruzzo production. Front. Microbio. 3:135. doi: 10.3389/fmicb.2012.00135

This article was submitted to Frontiers in Food Microbiology, a specialty of Frontiers in Microbiology.

Copyright () 2012 Suzzi, Schirone, Sergi, Marianella, Fasoli, Aguzzi and Tofalo. This is an open-access article distributed under the terms of the Creative Commons Attribution Non Commercial License, which permits non-commercial use, distribution, and reproduction in other forums, provided the original authors and source are credited. 